\title{
About disconnected topologies and synchronization of homogeneous nonlinear agents over switching networks
}

\author{
Giacomo Casadei, Alberto Isidori and Lorenzo Marconi
}

\begin{abstract}
In this paper we study the problem of networks on nonlinear oscillators when communication topologies are disconnected. In the first part we show that the high gain design procedure, proposed in [18], succeed in achieving clustered consensus when disconnected topologies occur. In the second part of the paper, we study a network of nonlinear oscillators when the topology is switching between a set of disconnected and connected topologies. By a Lyapunov analysis we prove that, if disconnected topologies last a limited amount of time and the switching law for connected topologies fulfill an average dwell time condition, synchronization is achieved.
\end{abstract}

\section{INTRODUCTION}

The problem of achieving consensus among a set of systems exchanging information through a network is extensively studied in the control literature (see [15] for an extensive survey of results in the field). Information network analysis, multi-agent systems, electrical power systems, animal collective behaviour, systems biology are just a few application domains where consensus among networked agents plays a role. One of the distinguishing elements of the framework where consensus problems are formulated is how the exchange of information between the agents is modeled. Graph theory is typically used in order to model communication networks, [25].

Many contributions about consensus usually require the graph to be connected and time invariant. These assumptions might be restrictive in many applications and thus motivated the analysis of disconnected topologies and switching networks.

The problem of disconnected topologies has been studied in order to understand if undesired or unstable behaviour might arise. Indeed, disconnected topologies typically lead to the so called clustering behaviour, in which agents achieve different synchronisation patterns, depending on connected sub-graphs composing the network. In [3], the clustering behaviour has been extensively studied and it is shown that this behaviour arises naturally in animal packs. This phenomenon poses challenging questions in terms of analysis and modelling of biological networks, especially when the number of agents interacting increases. A similar behaviour can be noticed also in opinion dynamics networks, where confidence is a time varying parameter based on which agents give trust only to a certain number of neighbors (see [4]).

Time varying topologies also attracted a lot of interests in the community and several results can be found. Among others, in [11], the problem of switching network is addressed and consensus is achieved as long as the graph is uniformly quasi-strongly connected and fulfills a dwell-time condition. For the special case of integrators network, [13] and [12] give a consensus result based on the concept of average connectivity of the graph. In [10] and [14], the problem of consensus of linear systems with switching topology is considered:results are provided respectively by means of a hybrid control techniques and small gain/passivity arguments. In [7], fully nonlinear agents are considered and sufficient conditions to achieve consensus are cast in terms of linear matrix inequalities and frequency of switching, taking into account also the impact of communication delay.

A very general framework proposed in literature to frame problems of switching networks is the one based on the notion of joint connectivity. In contrast with the definition of connected graphs, joint connectivity does not require the graph to be instantaneously connected. Rather, the union on a certain time interval of all topologies among which the network is switching is required to be connected. Within this general framework, in [5], [6] different consensus results (such as target aggregation and state agreement) are proved under the assumption that the graph is jointly strongly connected and that each topology persist for a time period fulfilling a dwell time condition. The agents dynamics are linear in [6] while nonlinear dynamics for "informed agents", fulfilling an attractivity condition to the target set, are considered in [5].

G. Casadei was with with the CASY - DEI, University of Bologna, now with Univ. Grenoble Alpes, CNRS, Inria, GIPSA-lab, F-38000 Grenoble, France. L. Marconi is with the CASY - DEI, University of Bologna. A. Isidori is with DIS-"Sapienza" - Università di Roma, Roma, Italy. giacomo. casadei@unibo.it, giacomo. casadei@gipsa-lab.grenoble-inp. fr. 
Recently, the analysis developed in [6] has been extended in [8] to the case of nonlinear agents. In this work a general class of nonlinear systems assumed to be "non-expansive" is considered, and strong joint connectivity of the graph is shown to be sufficient condition for consensus. In the same paper it is shown that if the nonlinear dynamics just fulfill a globally Lipschitz condition then consensus is achieved under the assumption that graph is uniformly strongly joint connected and the Lipschitz constant of the nonlinear dynamics is sufficiently small. However, assuming that the graph is uniformly strongly connected implies that all agents are centroid of the graph. Furthermore, to achieve state synchronization, the resulting average-graph is supposed to be fixed. These two conditions limit enormously the family of possible topology and the switching law sequence.

Another perspective on the problem on switching networks of nonlinear agents was introduced in [9] where the authors consider the switching law as a design parameter and, by means of switched systems tools, they show that a design it is possible to achieve synchronization as long as the switching law fulfills conditions equivalent to uniform joint connectivity.

In this work, by means of Lyapunov analysis, we describe how disconnected topologies affect the behaviour of the agents and that, following the design procedure proposed in [18], a clustering behavior arises naturally even in the nonlinear framework.

With respect to switching networks, we extend the results of [20], by considering the case in which the communication graph switches within a set of topologies not all necessarily connected and with time intervals in which connected topologies do not fulfill a dwell-time condition. More specifically, we show that consensus can be achieved provided that the time intervals in which the communication topologies are connected fulfill an average dwell-time condition ([22]). With respect to the works on the subject mentioned before, the emphasis of the paper is to deal with the lack of a guaranteed dwell-time. Lyapunov arguments proposed in the field of hybrid control systems (see [1], [23]) are used to prove the main result. This work frames as an addendum of [18] in which heterogeneous systems networked with fixed connected topologies are considered. In particular, the arguments presented here can be used to show that the control framework of [18] succeeds in achieving consensus between networked heterogeneous nonlinear systems even in presence of switching topologies of the kind considered in the following.

The paper is structured as follows. In Section II, we provide standard concepts about graph theory and define the framework of the note. In Section III, we study a network of nonlinear oscillators when disconnected topologies occur. In Section IV, starting from the results in previous section, we analyze the switching network scenario and provide sufficient conditions for synchronization achievement.

\section{BASIC FACTS AND FRAMEWORK}

\section{A. Graph Theory and Analysis}

The paper deals with the problem of reaching a consensus among a set of $N_{\mathrm{a}}$ agents, described by homogeneous nonlinear systems. Denoting by $\mathcal{V}=\left\{v_{1}, v_{2}, \ldots, v_{N_{\mathrm{a}}}\right\}$ the set of $N_{\mathrm{a}}$ nodes of the network, a topology is described by a directed communication graph given by the following objects:

- $\mathcal{E} \subset \mathcal{V} \times \mathcal{V}$ is a set of edges that models the interconnection between nodes, according to the following convention: $\left(v_{k}, v_{j}\right)$ belongs to $\mathcal{E}$ if there is a flow of information from node $j$ to node $k$. It is assumed that there are no self-loops, i.e. that $\left(v_{k}, v_{k}\right) \notin \mathcal{E}$.

- for each $\left(v_{k}, v_{j}\right) \in \mathcal{E}$ the flow of information from node $j$ to node $k$ in the $i$-th topology is weighted by the $(k, j)$-th entry $a_{k j} \geq 0$ of the so-called adjacency matrix $A \in \mathbb{R}^{N_{\mathrm{a}} \times N_{\mathrm{a}}}$.

The Laplacian matrix $L$ associated to the graph can be also immediately computed from the adjacency matrix $A$ in the usual way. In particular, $L$ is the $N_{\mathrm{a}} \times N_{\mathrm{a}}$ real matrix whose $(k, j)$-th entry $\ell_{k j}$ is defined as

$$
\begin{array}{ll}
\ell_{k j}(t)=-a_{k j} & \text { for } k \neq j \\
\ell_{k j}(t)=\sum_{m=1}^{N_{\mathrm{a}}} a_{k m} & \text { for } k=j .
\end{array}
$$

By definition, the diagonal entries of $L$ are non-negative, the off-diagonal entries are non-positive and, for each row, the sum of all entries on this row is zero (namely $L$ is a Metzler matrix). 
Theorem 1 A time-invariant graph, whose Laplacian matrix $L$ is Metzler, is connected if and only if $L$ has only one trivial eigenvalue $\lambda_{1}=0$ and all other eigenvalues $\lambda_{2}(L), \ldots, \lambda_{N}(L)$ have positive real parts.

To analyze the behvior of the network when disconnected topologies occur, we recall some useful concepts (see [15] and [19]). We start by recalling the definition of an independent connected component (ICC) of a di-graph $\mathcal{G}=\{\mathcal{V}, \mathcal{E}, A\}$.

Definition 1 An independent connected component of a di-graph $\mathcal{G}=\{\mathcal{V}, \mathcal{E}, A\}$ is the maximal subgraph $\tilde{\mathcal{G}}=$ $\{\tilde{\mathcal{V}}, \tilde{\mathcal{E}}, \tilde{A}\}$ that is connected and such that there is no edge in $\mathcal{E}$ with a tail outside $\tilde{\mathcal{V}}$ and the head in $\tilde{\mathcal{V}}$.

Let $c \geq 1$ be the number of $I C C$ s of the di-graph. We can partition the whole graph in $c+1$ sub-graphs: the first $c$ sub-graphs represent the ICCs inside the graph. The additional one is a "residual graph" (possibly empty) composed by "residual vertexes" that might have incoming edges from the independent connected components but without outgoing arcs toward independent connected components.

By relabeling the vertexes of the whole graph, so that the vertexes of each independent connected components are consecutive and the residual sub-graph vertexes are confined at the end, it turns out that the Laplacian matrix takes the form

$$
L=\left(\begin{array}{ccccc}
L_{1} & 0 & \cdots & 0 & 0 \\
0 & L_{2} & \cdots & 0 & 0 \\
\cdots & \cdots & \cdots & \cdots & \cdots \\
0 & 0 & \cdots & L_{c} & 0 \\
& & \Gamma & & L_{\text {res }}
\end{array}\right)
$$

where $\Gamma$ denotes the matrix of incoming edges to the residual component.

We observe that the eigenvalues of the Laplacians $L_{1}, \ldots, L_{c}$ are one zero and the rest are positive. It turns out that the eigenvalues of $L_{r e s}$ are all positive and thus $L_{r e s}$ is always invertible ${ }^{1}$. This fact is a necessary condition for the next proposition (whose proof can be found in [19]), in which $N_{1}, \ldots, N_{c}$, and $N_{\text {res }}$ denote the number of vertexes in each of the $c$ independent connected components.

Lemma 1 Let $V \in \mathbb{R}^{N \times c}$ be the matrix defined as

$$
V=\left(\begin{array}{cccc}
1_{N_{1} \times 1} & 0 & \cdots & 0 \\
0 & 1_{N_{2} \times 1} & \cdots & 0 \\
\cdots & \cdots & \cdots & \cdots \\
0 & 0 & \cdots & 1_{N_{c} \times 1} \\
\gamma_{1} & \gamma_{2} & \cdots & \gamma_{c}
\end{array}\right)
$$

with $\left[\gamma_{1}, \gamma_{2}, \ldots, \gamma_{c}\right]$ defined as

$$
\left[\gamma_{1}, \gamma_{2}, \ldots, \gamma_{c}\right]=-L_{\text {res }}^{-1} \Gamma\left(\begin{array}{cccc}
1_{N_{1} \times 1} & 0 & \ldots & 0 \\
0 & 1_{N_{2} \times 1} & \ldots & 0 \\
\ldots & \ldots & \ldots & \ldots \\
0 & 0 & \ldots & 1_{N_{c} \times 1}
\end{array}\right)
$$

where $\left(\begin{array}{ll}\Gamma & L_{\text {res }}\end{array}\right)$ denotes the matrix obtained by extracting the last $N_{\text {res }}$ rows of $L$. Then the following holds:

- $\operatorname{dim}(\operatorname{Ker}(L))=c$;

- the columns of $V$ form a basis of $\operatorname{Ker}(L)$.

With the previous result in hand, reminding that $N_{i}$ is the dimension of the $i$-th $I C C$, we define the matrix

$$
\Lambda_{i}=\left[\begin{array}{c}
0 \\
1 \times N_{i}-1 \\
I_{N_{i}-1}
\end{array}\right]
$$

${ }^{1}$ For the proof, see [15] Appendix A.3.2, proof of theorem 2.13. 
and consequently the transformation $T$

$$
T=\left[\begin{array}{cccccc}
1_{N_{1} \times 1} & \Lambda_{1} & 0 & \ldots & 0 & 0 \\
0 & 0 & & & \vdots & \vdots \\
\vdots & \vdots & \ddots & & 0 & 0 \\
0 & 0 & & 1_{N_{c} \times 1} & \Lambda_{c} & 0 \\
\gamma_{1} & 0 & \ldots & \gamma_{c} & 0 & I_{\text {res }}
\end{array}\right]
$$

where all the 0's blocks are defined according to the dimension of each ICC.

By applying such a transformation to $L$ we obtain $\tilde{L}=T^{-1} L T$ which, by reordering opportunely its columns, has the following structure

$$
\tilde{L}=\left[\begin{array}{ccc}
0_{c \times c} & L_{12} & 0_{c \times N_{\text {res }}} \\
0_{N-c-N_{\text {res }} \times 1} & L_{22} & 0_{N-c-N_{\text {res }} \times N_{\text {res }}} \\
0_{N_{\text {res }} \times c} & \tilde{\Gamma} & \tilde{L}_{\text {res }}
\end{array}\right]
$$

for some appropriately defined $L_{12}, L_{22}, \tilde{\Gamma}, \tilde{L}_{r e s}$, with all the eigenvalues of $L_{22}$ and $\tilde{L}_{r e s}$ that are positive.

By opportunely relabeling the vertexes, it turns out that $L_{12}=\operatorname{blkdiag}\left(L_{12_{1}}, \ldots, L_{12_{c}}\right)$ and $L_{22}=\operatorname{blkdiag}\left(L_{22_{1}}, \ldots, L_{22_{c}}\right)$.

Remark 1 It is worth noting that, under the condition that the spectrum of $L_{r e s}$ and $L_{22}$ are disjoint, namely

$$
\sigma\left(L_{22}\right) \cap \sigma\left(L_{\text {res }}\right)=\varnothing
$$

it is possible to define the transformation $T$ in (1) in such a way that $\tilde{\Gamma}=0$ (see [32] for more details). If this is the case, the matrix $\tilde{L}$ would turn out to be

$$
\tilde{L}=\left[\begin{array}{ccc}
0_{c \times c} & L_{12} & 0_{c \times N_{\text {res }}} \\
0_{N-c-N_{\text {res }} \times 1} & L_{22} & 0_{N-c-N_{\text {res }} \times N_{\text {res }}} \\
0_{N_{\text {res }} \times c} & 0_{N_{\text {res }} \times N-c-N_{\text {res }}} & \tilde{L}_{\text {res }}
\end{array}\right]
$$

The hypothesis that the spectrum of $L_{r e s}$ and $L_{22}$ are disjoint is often too restrictive. Hence, for the sake of generality, in the rest of the paper we will consider the case in which $\tilde{\Gamma} \neq 0$.

\section{B. Framework and main assumptions}

Each of the $N_{\mathrm{a}}$ agents is described by the nonlinear dynamics

$$
\begin{aligned}
\dot{x}_{k} & =f\left(x_{k}\right)+u_{k} \quad x_{k} \in \mathbb{R}^{d} \\
\iota_{k} & =h\left(x_{k}\right)
\end{aligned}
$$

in which, for each $k=1, \ldots, N_{\mathrm{a}}, u_{k} \in \mathbb{R}^{d}$ is the control input, $\iota_{k} \in \mathbb{R}$ is the available measurement. Note that we deal with homogeneous nonlinear agents, namely $f(\cdot)$ and $h(\cdot)$ do not depend on $k$.

We look for a decentralized control structure in which the agents exchange only output information and the control law of each agent is taken as

$$
u_{k}=\mathcal{K}\left(x_{k}\right) \nu_{k}, \quad \nu_{k}=\sum_{j=1}^{N_{\mathrm{a}}} \ell_{k j} h\left(x_{j}\right)
$$

with $\mathcal{K}\left(x_{k}\right)$ to be designed in such a way that output consensus is reached among the agents. Namely, for each initial condition $x_{k}(0) \in \mathbb{R}^{d}$, there is a function $\iota^{\star}: \mathbb{R} \rightarrow \mathbb{R}$ such that

$$
\lim _{t \rightarrow \infty}\left|\iota_{k}(t)-\iota^{\star}(t)\right|=0,
$$

uniformly in the initial conditions, for all $k=1, \ldots, N_{\mathrm{a}}$. It is worth noting that, in the proposed framework, no leader is considered, and only the neighbor's information is available according to the underlying communication topology. Furthermore, local output of single agents rather than a full state information is assumed to be spread over the network. 
The main results are proved under a certain number of assumptions regarding the agents that are here presented. Since the goal is to achieve consensus by only processing the output $\iota_{k}$ of each agent, the first assumption asks that systems (2) are uniformly observable (see [31]) as detailed next.

Assumption 1 The map $\Psi: \mathbb{R}^{d} \mapsto \mathbb{R}^{d}$ defined as

$$
\Psi\left(x_{k}\right)=\left(\begin{array}{c}
h\left(x_{k}\right) \\
L_{f} h\left(x_{k}\right) \\
\vdots \\
L_{f}^{d-1} h\left(x_{k}\right)
\end{array}\right)
$$

is a global diffeomorphism.

The requirement of the existence of a global diffeomorphism $\Psi(\cdot)$ is motivated by the fact that, in the following, we look for consensus results that hold globally, namely without restrictions on the initial state of the agents. The previous assumption could be weakened by just asking that the map $\Psi(\cdot)$ is a local diffeomorphism on some given set at the price of obtaining just semiglobal consensus results, namely by restricting the initial state of the agents to some prescribed compact set. Details in this direction are omitted since they can be obtained by using tools that are customary in the literature of stabilization of nonlinear systems.

The existence of such a diffeomorphism allows us to define a change of coordinate $w_{k}=\Psi\left(x_{k}\right)$, which maps system (2) to

$$
\dot{w}_{k}=S w_{k}+B \phi\left(w_{k}\right)+v_{k}, \quad y_{k}=C w
$$

with

$$
v_{k}=\frac{\mathrm{d} \Psi\left(x_{k}\right)}{\mathrm{d} x_{k}} u_{k}
$$

and with the triplet of matrices $(S, B, C)$ that is in prime form, that is $S$ is a shift matrix (all 1's on the upper diagonal and all 0 's elsewhere), $B^{T}=\left(\begin{array}{lll}0 \cdots 0 & 1\end{array}\right)$ and $C=\left(\begin{array}{lll}1 & 0 \cdots 0\end{array}\right)$. In the following we assume that the function

$$
\phi\left(w_{k}\right)=\left.L_{\mathrm{f}}^{d} h\left(x_{k}\right)\right|_{x_{k}=\Psi^{-1}\left(w_{k}\right)}
$$

is globally Lipschitz, namely there exists a positive constant $\bar{\phi}$ such that $\|\phi(w)\| \leq \bar{\phi}\|w\|$ for all $w \in \mathbb{R}^{d}$. Such a globally Lipschitz condition is motivated by the fact of looking for "global" consensus results. The assumption in question could be weakened by just asking the previous function to be only locally Lipschitz if just semiglobal consensus results are of interest.

By bearing in mind the definition of (5), we design $\mathcal{K}\left(x_{k}\right)$ in (3) as

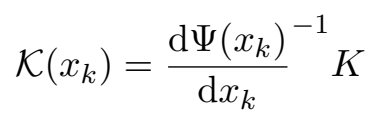

and thus obtain

$$
v_{k}=-K \sum_{j=1}^{N_{\mathrm{a}}} \ell_{k j} C w_{j} \quad k=1, \ldots, N_{\mathrm{a}} .
$$

We also assume that agents (5) have a robust compact attractor $W \subset \mathbb{R}^{d}$, where robustness is characterized in terms of Input-to-State Stability. This assumptions indeed guarantees that the network of (5) achieves synchronization on non-trivial trajectories.

Assumption 2 There exists a compact set $W \subset \mathbb{R}^{d}$ invariant for (2) with $u=0$ such that the system

$$
\dot{w}=S w+B \phi(w)+u
$$


is input-to-state stable with respect to $u$ relative to $W$, namely there exist a class- $\mathcal{K} \mathcal{L}$ function $\beta(\cdot, \cdot)$ and a class- $\mathcal{K}$ function $\gamma(\cdot)$ such that ${ }^{2}$

$$
\|w(t, \bar{w})\|_{W} \leq \max \left\{\beta\left(\|\bar{w}\|_{W}, t\right), \gamma\left(\sup _{\tau \in[0, t)}\|u(\tau)\|\right)\right\} .
$$

A relevant application scenario in which agents are described by nonlinear dynamics of the form of (5) is the one of power networks (see [27],[30]), where nonlinear oscillators are used to model nonlinear components of the electrical networks. A further motivation for considering agents described by (5) comes from the theory of output regulation for networked systems ( see [18], for details on synchronization for heterogeneous nonlinear networks), in which nonlinear oscillators of the kind of (5) have to be synchronized.

Finally, we fix a restriction on the communication topologies asking that the real part of the nontrivial eigenvalues of the Laplacian are uniformly bounded from below by a known constant $\mu$.

Assumption 3 There exists a $\mu>0$ such that, for all $m=1, \ldots, N_{\mathrm{a}}$ such that $\lambda_{m}(L) \neq 0$, the following holds

$$
\operatorname{Re} \lambda_{m}(L) \geq \mu
$$

\section{ANALYSiS IN CASE OF DISCONNECTED TOPOLOGY}

In this section, we show that the control structure proposed in [18] does not affect the stability of the networked system, even if the communication topology is disconnected. In particular we will show that agents belonging to one of the independent connected components achieve consensus and the residual agents have bounded trajectories.

Following the design procedure in [18], we choose the vector $K$ in (7) as

$$
K=D_{g} K_{0}
$$

where $D_{g}=\operatorname{diag}\left(g, g^{2}, \ldots, g^{d}\right)$ with $g$ a high-gain parameter, and $K_{0}$ chosen as

$$
K_{0}=P C^{T}
$$

with $P$ solution of the algebraic Riccati equation

$$
S P+P S^{T}-2 \mu P C^{T} C P+a I=0
$$

with $a>0, S$ and $C$ as in (5) and $\mu$ as in Assumption 3.

Proposition 1 For all $x_{k}(0) \in \mathbb{R}^{d}, k=1, \ldots, N_{\mathrm{a}}$, the trajectories of the agents are bounded. Furthermore, agents belonging to a independent connected component achieve consensus, namely there is a function $\iota_{i}^{\star}: \mathbb{R} \rightarrow \mathbb{R}$, for $i=1, \ldots, c$, such that

$$
\lim _{t \rightarrow \infty}\left|\iota_{k}(t)-\iota_{i}^{\star}(t)\right|=0
$$

for every $k$ such that the $k$-th agent belongs to the $i$-th independent connected component.

Proof: The proof is divided in two parts. In the analysis of disconnected topologies, we will first focus on the independent connected components and show that inside each one of them consensus is achieved. Second, we will show that agents belonging to the residual component do not achieve consensus have bounded trajectories.

\footnotetext{
${ }^{2}$ Here and in the following we denote by $\|w\|_{W}=\min _{x \in W}\|w-x\|$ the distance of $w$ from $W$. Furthermore, $w(t, \bar{w})$ denotes the solution of (2) at time $t$ with initial condition $\bar{w}$ at time $t=0$.
} 


\section{A. Consensus inside independent connected components}

By grouping all the agents according to $\mathbf{w}=\operatorname{col}\left(w_{1}, \ldots, w_{N}\right)$ and considering a fixed disconnected topology, we change coordinate according to $\mathbf{z}=\left(T^{-1} \otimes I_{d}\right) \mathbf{w}$, where $T$ is defined in (1) relatively to a generic topology.

By relabeling the agents so that the agents within the same independent connected component are consecutive and the residual agents are confined at the end, $\mathbf{z}$ turns out to be

$$
\mathbf{z}=\operatorname{col}\left(\operatorname{col}\left(z_{11}, \mathbf{z}_{12}\right), \ldots, \operatorname{col}\left(z_{c 1}, \mathbf{z}_{c 2}\right), \mathbf{z}_{r e s}\right)
$$

with $z_{i 1} \in \mathbb{R}^{d}$ and $\mathbf{z}_{i 2} \in \mathbb{R}^{d\left(N_{i}-1\right)}$, for $i=1, \ldots, c$ and $\mathbf{z}_{r e s} \in \mathbb{R}^{d N_{\text {res }}}$.

The agents belonging to the $c$ connected components are described by

$$
\begin{aligned}
\dot{z}_{j_{1}} & =S z_{j_{1}}+B \phi\left(z_{j_{1}}\right)-\left(L_{12_{j}} \otimes K C\right) \mathbf{z}_{j_{2}} \\
\dot{\mathbf{z}}_{j_{2}} & =\left(\left[I_{N_{j}-1} \otimes S\right]-\left[L_{22_{j}} \otimes K C\right]\right) \mathbf{z}_{j_{2}}+\Delta \Phi_{j}\left(z_{j_{1}}, \mathbf{z}_{j_{2}}\right)
\end{aligned}
$$

for $j=1, \ldots, c, z_{j_{1}} \in \mathbb{R}^{d}$ and $\mathbf{z}_{j_{2}} \in \mathbb{R}^{d\left(N_{j}-1\right)}$, with $\Delta \Phi_{j}\left(z_{j_{1}}, \mathbf{z}_{j_{2}}\right)$

$$
\Delta \Phi_{j}\left(z_{j_{1}}, \mathbf{z}_{j_{2}}\right)=\left(I_{N_{j}-1} \otimes B\right)\left(\begin{array}{c}
\phi\left(z_{j_{1}}+z_{j_{2}}\right)-\phi\left(z_{j_{1}}\right) \\
\vdots \\
\left.\phi\left(z_{j_{1}}+z_{j_{N_{i}}}\right)-\phi\left(z_{j_{1}}\right)\right)
\end{array}\right)
$$

which is globally Lipschitz in $\mathbf{z}_{j_{2}}$ uniformly in $z_{j_{1}}$ and $\Delta \Phi_{j}\left(z_{j_{1}}, 0\right)=0$. As proposed in [18], we now rescale the variable $\mathbf{z}_{j_{2}}$ in the following way

$$
\chi_{j}=\left(I_{N_{j}-1} \otimes D_{g}^{-1}\right) \mathbf{z}_{j_{2}}
$$

and obtain

$$
\begin{aligned}
& \dot{z}_{j_{1}}=S z_{j_{1}}+B \phi\left(z_{j_{1}}\right)-\left(L_{12_{j}} \otimes D_{g} K_{0} C\right)\left(I_{N_{\mathrm{a}}-1} \otimes D_{g}\right) \chi \\
& \dot{\chi}_{j}=g H_{j} \chi_{j}+\frac{1}{g^{d}} \Delta \Phi\left(z_{j_{1}},\left(I_{N_{\mathrm{a}}-1} \otimes D_{g}\right) \chi_{j}\right)
\end{aligned}
$$

where $H_{j}=\left[\left(I_{N_{j}-1} \otimes S\right)-\left(L_{22_{j}} \otimes K_{0} C\right)\right]$. To show that the origin of the system with state $\chi_{j}$ is locally asymptotically stable, we consider the change of variable $\zeta_{j}=J_{j} \chi_{j}$ with $J_{j}$ such that $\bar{H}_{j}=J_{j} H_{j} J_{j}^{-1}$ is in Jordan form. We obtain a new system that is the cascade of system

$$
\dot{\zeta}_{j}=g \bar{H}_{j} \zeta_{j}+\frac{1}{g^{d}} \Delta \Phi\left(z_{j_{1}},\left(I_{N_{\mathrm{a}}-1} \otimes D_{g}\right) J_{j}^{-1} \zeta_{j}\right)
$$

with system

$$
\dot{z}_{j_{1}}=S z_{j_{1}}+B \phi\left(z_{j_{1}}\right)-\left(L_{i, 12} \otimes D_{g} K_{0} C\right)\left(I_{N_{\mathrm{a}}-1} \otimes D_{g}\right) J_{i}^{-1} \zeta_{j}
$$

To prove that consensus is achieved inside each of the $c$ independent connected components $(i . e . j=1, \ldots, c)$, we now use Lypunov arguments. For the sake of simplicity in the notation, we drop the dependence on $j$. Consider the candidate Lyapunov function

$$
V(\zeta)=\zeta^{T}\left(D(\ell) \otimes P^{-1}\right) \zeta
$$

where $P$ is the solution of $(10)$ and $D(\ell)=\operatorname{diag}\left(1, \ell, \ell^{2}, \ldots, \ell^{N_{\mathrm{a}}-2}\right)$ with $\ell$ a positive design parameter yet to be fixed. Note that there exist positive constants $\underline{\lambda} \leq \bar{\lambda}$, both dependent on $\ell$, such that $\underline{\lambda} \zeta^{T} \zeta \leq V \leq \bar{\lambda} \zeta^{T} \zeta$.

The derivative of $V$ along the solutions of (20) can be bounded as

$$
\begin{aligned}
\dot{V} & =2 \zeta^{T}\left(D(\ell) \otimes P^{-1}\right)\left[g \bar{H} \zeta+\frac{1}{g^{d}} \Delta \Phi\left(z_{1},\left(I_{N_{a}-1} \otimes D_{g}\right) J_{i}^{-1} \zeta\right)\right] \\
& \leq 2 \zeta^{T}\left(D(\ell) \otimes P^{-1}\right) g \bar{H} \zeta+\frac{2}{g^{d}} \bar{\Phi}\left\|D(\ell) \otimes P^{-1}\right\|\left\|\left(I_{N_{\mathrm{a}}-1} \otimes D_{g}\right)\right\|\left\|J_{i}^{-1}\right\| \zeta^{T} \zeta \\
& \leq 2 \zeta^{T}\left(D(\ell) \otimes P^{-1}\right) g \bar{H} \zeta+a_{\phi} \zeta^{T} \zeta
\end{aligned}
$$

where $a_{\phi}$ is a positive constant not dependent on $g$ (provided that the latter is taken $g \geq 1$ ).

From Theorem 1, we know that that if the graph is connected one eigenvalues of a Laplacian matrix is zero and the rest are all positive. We recall this crucial result (see [18]). 
Lemma 2 Let Assumption 2 hold. Then, for each of the c independent connected component inside the graph, there exist a positive constants $a_{\mathrm{c}}^{\prime}$ and $\ell^{\star}$ such that for all $\ell \geq \ell^{\star}$

$$
2 \zeta^{T}\left(D(\ell) \otimes P^{-1}\right) \bar{H} \zeta \leq-a_{\mathrm{c}}^{\prime} \zeta^{T} \zeta .
$$

Using the previous lemma and taking $g^{\star}=\left(a_{\phi}+a_{\mathrm{c}} \bar{\lambda}\right) / a_{\mathrm{c}}^{\prime}$ with $a_{\mathrm{c}}$ an arbitrary positive constant $\left(g^{\star} \geq 1\right.$ without loss of generality), it is immediately seen that for all $\ell \geq \ell^{\star}$ and $g \geq g^{\star}$ we have

$$
\dot{V} \leq-\left(g a_{\mathrm{c}}^{\prime}-a_{\phi}\right) \zeta^{T} \zeta \leq-\frac{g a_{\mathrm{c}}^{\prime}-a_{\phi}}{\bar{\lambda}} V \leq-a_{\mathrm{c}} V .
$$

By this we conclude that consensus is achieved within each cluster. It is worth noting that if the graph underlying the communication is connected, i.e. $c=1$, the previous analysis shows that synchronization of the whole network is achieved.

\section{B. Behavior of the residual agents}

The residual subsystem, instead, reads as

$$
\dot{\mathbf{z}}_{r e s}=\left(\left[I_{N_{\text {res }}} \otimes S\right]-\left[\tilde{L}_{r e s} \otimes K C\right]\right) \mathbf{z}_{r e s}+\Delta \Phi_{\text {res }}\left(z_{1_{1}}, \ldots, z_{c_{1}}, \mathbf{z}_{r e s}\right)+(\tilde{\Gamma} \otimes K C) \tilde{\mathbf{z}}_{2}
$$

with $\tilde{\mathbf{z}}_{2}=\operatorname{col}\left(\mathbf{z}_{1_{2}}, \ldots, \mathbf{z}_{c_{2}}\right)$ and where $\Delta \Phi_{r e s}\left(z_{1_{1}}, \ldots, z_{c_{1}}, \mathbf{z}_{r e s}\right)$ is

$$
\left(I_{N_{\text {res }}} \otimes B\right)\left(\begin{array}{c}
-\psi_{1_{1}} \phi\left(z_{1_{1}}\right)-\ldots-\psi_{c_{1}} \phi\left(z_{c_{1}}\right)+\phi_{r e s_{1}} \\
\vdots \\
-\psi_{1_{N_{r e s}}} \phi\left(z_{1_{1}}\right)-\ldots-\psi_{c_{N_{r e s}}} \phi\left(z_{c_{1}}\right)+\phi_{r e s_{N_{r e s}}}
\end{array}\right)
$$

where $\phi_{\text {res }_{1}}, \ldots, \phi_{\text {res }_{c}}$ are defined as

$$
\begin{gathered}
\phi_{r e s_{1}}=\phi\left(\psi_{1_{1}} z_{1_{1}}+\ldots+\psi_{c_{1}} z_{c_{1}}+z_{r e s_{1}}\right) \\
\vdots \\
\phi_{r e s_{c}}=\phi\left(\psi_{1_{N_{r e s}}} z_{1_{1}}+\ldots+\psi_{c_{N_{r e s}}} z_{c_{1}}+z_{r e s_{N_{r e s}}}\right) .
\end{gathered}
$$

Note that, in general, $\Delta \Phi_{\text {res }}\left(z_{1_{1}}, \ldots, z_{c_{1}}, 0\right) \neq 0$.

We now change coordinate according to $\zeta_{\text {res }}=\left(I_{N_{\text {res }}} \otimes D_{g}^{-1}\right) \mathbf{z}_{\text {res }}$ and obtain

$$
\dot{\zeta}_{\text {res }}=g\left(\left[I_{N_{\text {res }}} \otimes S\right]-\left[\tilde{L}_{\text {res }} \otimes K_{0} C\right]\right) \zeta_{\text {res }}+\frac{1}{g^{d}} \Delta \Phi_{r e s}\left(z_{1_{1}}, \ldots, z_{c_{1}},\left(I_{N_{\text {res }}} \otimes D_{g}\right) \zeta_{\text {res }}\right)+\left(\tilde{\Gamma} \otimes K_{0} C\right) \tilde{\mathbf{z}}_{2}
$$

The fact that $\tilde{L}_{\text {res }}$ has positive eigenvalues leads to conclude that the matrix

$$
\left[I_{N_{\text {res }}} \otimes S\right]-\left[\tilde{L}_{r e s} \otimes K C\right]=H_{\text {res }}
$$

is Hurwitz. Furthermore, by adding and subtracting the term $\Delta \Phi_{r e s}\left(z_{1_{1}}, \ldots, z_{c_{1}}, 0\right)$, we obtain

$$
\begin{aligned}
\dot{\zeta}_{\text {res }} & =g H_{\text {res }} \zeta_{\text {res }}+\frac{1}{g^{d}} \Delta \Phi_{r e s}\left(z_{1_{1}}, \ldots, z_{c_{1}},\left(I_{N_{\text {res }}} \otimes D_{g}\right) \zeta_{\text {res }}\right)+\left(\tilde{\Gamma} \otimes K_{0} C\right) \tilde{\mathbf{z}}_{2} \pm \frac{1}{g^{d}} \Delta \Phi_{r e s}\left(z_{1_{1}}, \ldots, z_{c_{1}}, 0\right) \\
& =g H_{\text {res }} \zeta_{\text {res }}+\frac{1}{g^{d}} \Delta \Phi_{r e s}\left(z_{1_{1}}, \ldots, z_{c_{1}}, 0\right)+\left(\tilde{\Gamma} \otimes K_{0} C\right) \tilde{\mathbf{z}}_{2}+\frac{1}{g^{d}} \Delta \tilde{\Phi}_{r e s}\left(z_{1_{1}}, \ldots, z_{c_{1}},\left(I_{N_{\text {res }}} \otimes D_{g}\right) \zeta_{\text {res }}\right)
\end{aligned}
$$

where

$$
\Delta \tilde{\Phi}(\cdot)=\Delta \Phi_{\text {res }}\left(z_{1_{1}}, \ldots, z_{c_{1}},\left(I_{N_{\text {res }}} \otimes D_{g}\right) \zeta_{\text {res }}\right)-\Delta \Phi_{\text {res }}\left(z_{1_{1}}, \ldots, z_{c_{1}}, 0\right)
$$

It is is easy to see that, $\zeta_{\text {res }}=0$ implies $\Delta \tilde{\Phi}\left(z_{1_{1}}, \ldots, z_{c_{1}}, 0\right)=0$. From this and the fact that both $\Delta \Phi_{\text {res }}\left(z_{1_{1}}, \ldots, z_{c_{1}}, 0\right)$ and $\left(\tilde{\Gamma} \otimes K_{0} C\right) \tilde{\mathbf{z}}_{2}$ are bounded since $z_{1_{1}}, \mathbf{z}_{1_{2}}, \ldots, z_{c_{1}}, \mathbf{z}_{c_{2}}$ are all bounded (as shown in the first part of the proof), we can conclude that, for a sufficiently large $g, \zeta_{\text {res }}$ is bounded and thus $\mathbf{z}_{r e s}$ is bounded too, independently from the particular topology.

Following the definition of (1), the fact that $\mathbf{z}_{r e s}$ is bounded and that all the agents belonging to an independent connected components achieve consensus, leads to conclude that $\mathbf{w}$ is bounded too, despite the particular disconnected topology. 


\section{Analysis of Switching Networks}

In this section, we present conditions under which consensus is achieved if the topology switches between a set of connected and disconnected topologies. First we will define the switching topology conditions and then, give the main result of this section.

\section{A. Switching topology framework}

We denote by $\mathcal{T}=\left\{\mathcal{T}_{1}, \ldots, \mathcal{T}_{N_{\mathrm{t}}}\right\}$ the set of $N_{\mathrm{t}}$ possible communication topologies. This set of topologies $\mathcal{T}$ is also characterized by topologies that are not necessarily connected ${ }^{3}$. For this reason we split the set $\mathcal{T}$ in two disjoint sets $\mathcal{T}_{\mathrm{c}}$ and $\mathcal{T}_{\text {nc }}$, which fulfill $\mathcal{T}=\mathcal{T}_{\mathrm{c}} \cup \mathcal{T}_{\text {nc }}$ and $\mathcal{T}_{\mathrm{c}} \cap \mathcal{T}_{\text {nc }}=\emptyset$, collecting topologies that are, respectively, connected and disconnected.

For all $i=1, \ldots, N_{\mathrm{t}}$, let $\Lambda_{i}=\left\{\lambda_{1}\left(L^{i}\right), \ldots, \lambda_{N_{\mathrm{a}}}\left(L^{i}\right)\right\}$ be the eigenvalues of $L^{i}$ (the Laplacian of the $i$-th topology), ordered with increasing real part. As a consequence of Theorem 1 and Lemma 1, the following holds (see [15] and [26] for further details):

- if $\mathcal{T}_{i} \in \mathcal{T}_{\mathrm{c}}$ then $\lambda_{1}\left(L^{i}\right)=0$ and $\operatorname{Re} \lambda_{m}\left(L^{i}\right)>0$ for $m=2, \ldots N_{\mathrm{a}}$;

- if $\mathcal{T}_{i} \in \mathcal{T}_{\text {nc }}$ then there exists a $c_{i} \in\left[1, N_{\mathrm{a}}\right]$ such that $\lambda_{m}\left(L^{i}\right)=0$ for $m=1 \ldots, c_{i}$ and $\operatorname{Re} \lambda_{m}\left(L^{i}\right)>0$ for $m=c_{i}+1 \ldots N_{\mathrm{a}}$.

The different communication topologies alternates in time by forming an ordered sequence $\left\{\mathcal{T}_{i}\right\}_{i=1}^{\infty}$, with each $\mathcal{T}_{i}$ taken in the set $\mathcal{T}$. We denote by $\Delta T_{i} \geq 0, i=1, \ldots, \infty$ the length of the time interval in which the $i$-th communication topology is active. Note that time intervals of zero length are allowed in the proposed framework. By this fact, without loss of generality, we can assume that the topologies alternates in time according to the rule that $\mathcal{T}_{i} \in \mathcal{T}_{\mathrm{c}}$ if $i$ is even and $\mathcal{T}_{i} \in \mathcal{T}_{\mathrm{nc}}$ is $i$ is odd. As a matter of fact, if two connected (disconnected) communication topologies occur in a row we can always "separate" them with a disconnected (connected) topology of zero length without practically changing the networked system dynamics. Note also that we do not assume that connected communication topologies persist for a guaranteed dwell time, namely connected topologies can last for arbitrarily small (indeed also of length zero) time interval. The kind of result we will prove (see next Proposition 1) is that consensus is reached if the intervals of time in which connected topologies governing the communication between the agents have a sufficiently long (in the average) duration and if the disconnected topologies duration is bounded.

We rewrite Assumption 3 in the switching topology framework in the following way.

Assumption 4 There exist a $\mu>0$ such that, for all $i=1, \ldots, N_{\mathrm{t}}$ and for all $m=1, \ldots, N_{\mathrm{a}}$ such that $\lambda_{m}\left(L^{i}\right) \neq 0$, the following holds

$$
\operatorname{Re} \lambda_{m}\left(L^{i}\right) \geq \mu
$$

We formulate now the assumption about the length of the time intervals in which disconnected topologies are active.

Assumption 5 There exists a $T_{0}>0$ such that for all $i=1, \ldots, N_{\mathrm{t}}$ such that $\mathcal{T}_{i} \in \mathcal{T}_{\mathrm{nc}}$ the following holds

$$
\Delta T_{i} \leq T_{0} .
$$

Remark 2 It is worth noticing that due to the results of Section III, since all the agents are bounded despite the particular disconnected topology, the parameter $T_{0}$ can be chosen arbitrarily big.

The additional condition under which the main result will be proved asks that the time intervals in which the network is connected last, in the average, sufficiently long. More precisely, we asks that there exist positive $\tau \in \mathbb{R}_{\geq 0}$ and $n_{0} \in \mathbb{N}$ such that, for all possible $n, i_{0} \in \mathbb{N}$ with $i_{0}$ even, we have

$$
\sum_{i=i_{0}, i=i+2}^{i_{0}+2 n} \Delta T_{i} \geq \tau\left(n-n_{0}\right)
$$

\footnotetext{
${ }^{3}$ We recall that a communication topology is said to be connected if there is a node $v$ from which any other node $v_{k} \in \mathcal{V} \backslash\{v\}$ can be reached, or equivalently if there is a path from $v$ to all $v_{k}$. In the previous definition a path from node $v_{j}$ to node $v_{k}$ in the $i$-th topology is a sequence of $r$ distinct nodes $\left\{v_{\ell_{1}}, \ldots, v_{\ell_{r}}\right\}$ with $v_{\ell_{1}}=v_{j}$ and $v_{\ell_{r}}=v_{k}$ such that $\left(v_{i+1}, v_{i}\right) \in \mathcal{E}_{i}$
} 
The previous condition can be regarded as a average dwell-time condition (see [22]), with the time $\tau$, in particular, that can be seen as an average length of the intervals in which the network is connected, and $n_{0}$ representing the number of "connected" intervals of zero duration that can occur in a row. The result formulated in the next proposition, in fact, claims that consensus is achieved if (16) is fulfilled for some $n_{0}$ and $\tau$ with the latter sufficiently large.

We conclude the section by remarking how the framework proposed in this paper to model switching graphs is, from one hand, more restrictive than the one based on the property of joint connectivity used, for instance, in [5], [6]. As a matter of fact, joint connectivity does not imply the existence of time intervals in which the graph is connected (as assumed in our framework) since it is the union of all possible network configurations that is required to have connectivity properties. On the other hand, all consensus results presented in literature that rely on a uniform joint connectivity condition ask that the different topologies persist a guaranteed dwell-time. In this respect the condition above, asking just a dwell-time in the average, is milder and much more general.

Furthermore, it is important to stress that in [5], [6] the graph is supposed to be uniformly strongly connected, which implies that all agents are centroid of the graph, while in our case we simply ask the topolgies to be connected. In addition, to achieve state synchronization, in [6], the graph is not only asked to be uniformly strongly connected but also to be fixed, a fact that imposes severe restrictions on the topology switching sequence.

\section{B. Main Result}

We now introduce the second result of the paper. We give the conditions under which the network of nonlinear agents achieve synchronization if the conditions detailed in previous sections are fulfilled.

Proposition 2 Consider the networked control system (5) controlled by (7) with $K$ as in (8) under the assumptions specified before and with the length of the time interval of connected topology fulfilling the average dwell-time condition (16) for some $n_{0} \geq 1$ and $\tau$. Then, there exist $a \tau^{\star}$ and $g^{\star}$ such that for all $\tau \geq \tau^{\star}$ and $g \geq g^{\star}$ the compact invariant set

$$
\mathbf{W}=\left\{\left(w_{1}, w_{2}, \ldots, w_{N_{\mathrm{a}}}\right) \in W \times W \times \cdots \times W: w_{1}=w_{2}=\cdots=w_{N_{\mathrm{a}}}\right\}
$$

is globally asymptotically stable for the closed-loop networked system. $\triangleleft$

Proof: The proof of Proposition 2 is divided in three sections:

- in the first part we consider the behavior of the network for a fixed connected topology and, by using the argument in the proof of Proposition 1, we introduce a common Lyapunov function proving consensus;

- in the second part we study the behavior of the network for a fixed disconnected topology by means of Lyapunov arguments;

- in the third and final part of the proof, we consider the network under switching topologies and, by means of hybrid Lyapunov tools, we prove that, under an average dwell time condition, the agents achieve consensus despite arbitrary long time intervals in which the network is not connected.

\section{Connected topologies}

This part of the proof follows arguments that are similar too the ones used in the proof of Proposition 1.

Consider a generic fixed topology $\mathcal{T}_{i} \in \mathcal{T}$ and the change of coordinate

$$
M=\left(\begin{array}{cc}
1 & 0_{1 \times\left(N_{\mathrm{a}}-1\right)} \\
1_{N_{\mathrm{a}}-1} & I_{N_{\mathrm{a}}-1}
\end{array}\right) .
$$

Defining $\mathbf{w}=\operatorname{col}\left(w_{1}, \ldots, w_{N_{\mathrm{a}}}\right)$ and by bearing in mind the choice of $K$, the networked system can be compactly rewritten as

$$
\dot{\mathbf{w}}=\left[\left(I_{N_{a}} \otimes S\right)-\left(L_{i} \otimes D_{g} K_{0} C\right)\right] \mathbf{w}+\left(I_{N_{a}} \otimes B\right) \Phi(\mathbf{w})
$$

where $\Phi(\mathbf{w})=\operatorname{col}\left(\phi\left(w_{1}\right), \ldots, \phi\left(w_{N_{\mathrm{a}}}\right)\right)$.

Elementary computations show that

$$
\tilde{L}^{i}=M^{-1} L^{i} M=\left(\begin{array}{cc}
0 & L_{12}^{i} \\
0_{\left(N_{\mathrm{a}}-1\right) \times 1} & L_{22}^{i}
\end{array}\right)
$$


where $L_{12}^{i}=L_{\left[1,2: N_{\mathrm{a}}\right]}^{i}$ and $L_{22}^{i}=L_{\left[2: N_{\mathrm{a}}, 2: N_{\mathrm{a}}\right]}^{i}-1_{N_{\mathrm{a}}-1} L_{\left[1,2: N_{\mathrm{a}}\right]}^{i}$. We consider now the change of variables

$$
\mathbf{w} \mapsto\left(\begin{array}{c}
\varrho_{1} \\
\varrho
\end{array}\right)=\left(M^{-1} \otimes I_{d}\right) \mathbf{w}
$$

with $\varrho_{1} \in \mathbb{R}^{d}$ and $\varrho \in \mathbb{R}^{\left(N_{\mathrm{a}}-1\right) d}$. Note that $\varrho_{1}=w_{1}$ and

$$
\varrho=\left(\begin{array}{llll}
w_{2}-w_{1} & w_{3}-w_{1} & \ldots & w_{N_{\mathrm{a}}}-w_{1}
\end{array}\right)^{T} .
$$

By using (19), an easy calculation shows that system (18) in the new coordinates reads as

$$
\begin{aligned}
\dot{\varrho}_{1} & =S \varrho_{1}+B \phi\left(\varrho_{1}\right)-\left(L_{12}^{i} \otimes D_{g} K_{0} C\right) \varrho \\
\dot{\varrho} & =\left[\left(I_{N_{\mathrm{a}}-1} \otimes S\right)-\left(L_{22}^{i} \otimes D_{g} K_{0} C\right)\right] \varrho+\Delta \Phi\left(\varrho_{1}, \varrho\right)
\end{aligned}
$$

where

$$
\Delta \Phi(\varrho 1, \varrho)=\left(I_{N-1} \otimes B\right)\left(\begin{array}{c}
\phi\left(\varrho_{2}+\varrho_{1}\right)-\phi\left(\varrho_{1}\right) \\
\vdots \\
\phi\left(\varrho_{N_{\mathrm{a}}}+\varrho_{1}\right)-\phi\left(\varrho_{1}\right)
\end{array}\right)
$$

where $\varrho=\operatorname{col}\left(\varrho_{2}, \ldots, \varrho_{N_{\mathrm{a}}}\right)$, with $\varrho_{i} \in \mathbb{R}^{d}, i=2, \ldots, N_{\mathrm{a}}$. Note that $\Delta \Phi\left(\varrho_{1}, \varrho\right)$ is globally Lipschitz in $\varrho$ uniformly in $\varrho_{1}$ and $\Delta \Phi\left(\varrho_{1}, 0\right)=0$ for all $\varrho_{1} \in \mathbb{R}^{d}$. Namely, there exists a positive $\bar{\Phi}$ such that $\left\|\Delta \Phi\left(\varrho_{1}, \varrho\right)\right\| \leq$ $\bar{\Phi}\|\varrho\|$ for all $\varrho_{1} \in \mathbb{R}^{d}$ and $\varrho \in \mathbb{R}^{\left(N_{\mathrm{a}}-1\right) d}$.

We now rescale the variable $\varrho$ in the following way

$$
\chi=\left(I_{N_{\mathrm{a}}-1} \otimes D_{g}^{-1}\right) \varrho
$$

and obtain

$$
\begin{aligned}
\dot{\varrho}_{1} & =S \varrho_{1}+B \phi\left(\varrho_{1}\right)-\left(L_{12}^{i} \otimes D_{g} K_{0} C\right)\left(I_{N_{\mathrm{a}}-1} \otimes D_{g}\right) \chi \\
\dot{\chi} & =g H_{i} \chi+\frac{1}{g^{d}} \Delta \Phi\left(z_{1},\left(I_{N_{\mathrm{a}}-1} \otimes D_{g}\right) \chi\right)
\end{aligned}
$$

where $H_{i}=\left[\left(I_{N-c} \otimes S\right)-\left(L_{22}^{i} \otimes K_{0} C\right)\right]$. To show that the origin of the system with state $\chi$ is locally asymptotically stable, we consider the change of variable $\zeta=J_{i} \chi$ with $J_{i}$ such that $\bar{H}_{i}=J_{i} H_{i} J_{i}^{-1}$ is in Jordan form. We obtain a new system that is the cascade of system

$$
\dot{\zeta}=g \bar{H}_{i} \zeta+\frac{1}{g^{d}} \Delta \Phi\left(z_{1},\left(I_{N_{\mathrm{a}}-1} \otimes D_{g}\right) J_{i}^{-1} \zeta\right)
$$

with system

$$
\dot{z}_{1}=S z_{1}+B \phi\left(z_{1}\right)-\left(L_{i, 12} \otimes D_{g} K_{0} C\right)\left(I_{N_{\mathrm{a}}-1} \otimes D_{g}\right) J_{i}^{-1} \zeta .
$$

We consider the candidate Lyapunov function

$$
V(\zeta)=\zeta^{T}\left(D(\ell) \otimes P^{-1}\right) \zeta
$$

where $P$ is the solution of $(10)$ and $D(\ell)=\operatorname{diag}\left(1, \ell, \ell^{2}, \ldots, \ell^{N_{\mathrm{a}}-2}\right)$ with $\ell$ a positive design parameter yet to be fixed. Note that there exist positive constants $\underline{\lambda} \leq \bar{\lambda}$, both dependent on $\ell$, such that $\underline{\lambda} \zeta^{T} \zeta \leq V \leq \bar{\lambda} \zeta^{T} \zeta$.

Using the results of Lemma 2 and taking $g^{\star}=\left(a_{\phi}+a_{\mathrm{c}} \bar{\lambda}\right) / a_{\mathrm{c}}^{\prime}$ with $a_{\mathrm{c}}$ an arbitrary positive constant $\left(g^{\star} \geq 1\right.$ without loss of generality), it is immediately seen that for all $\ell \geq \ell^{\star}$ and $g \geq g^{\star}$ we have

$$
\dot{V} \leq-\left(g a_{\mathrm{c}}^{\prime}-a_{\phi}\right) \zeta^{T} \zeta \leq-\frac{g a_{\mathrm{c}}^{\prime}-a_{\phi}}{\bar{\lambda}} V \leq-a_{\mathrm{c}} V
$$

Hence, (23) shows that when connected topologies occur, the Lyapunov function (22) is strictly decreasing along solution of (20). The local asymptotic stability of $\zeta$ and the ISS properties of $z_{1}$ (claimed in Assumption 2) guarantee that all the agents outputs $y_{k}$ move towards consensus on a common trajectory $y^{\star}$. 


\section{Disconnected topologies}

We consider now time intervals in which $\mathcal{T}_{i} \in \mathcal{T}_{\text {nc }}(i$ odd) and study the behavior of the common Lyapunov function (22). For all $i$ such that $\mathcal{T}_{i} \in \mathcal{T}_{\text {nc }}$, it comes straightforward that the matrix $\bar{H}_{i}$ in (20) is not Hurwitz. Hence the result in Lemma 2 cannot be claimed.

The derivative of (22) during time intervals in which the topology is not connected is

$$
\begin{aligned}
\dot{V} & =2 \zeta^{T}\left(D(\ell) \otimes P^{-1}\right)\left[g H_{i} \zeta+\frac{1}{g^{d}} \Delta \Phi\left(z_{1},\left(I_{N_{a}-1} \otimes D_{g}\right) \zeta\right)\right] \\
& \leq g a_{\mathrm{nc}}^{\prime} \zeta^{T} \zeta+\frac{2}{g^{d}} \bar{\Phi}\left\|D(\ell) \otimes P^{-1}\right\|\left\|\left(I_{N_{\mathrm{a}}-1} \otimes D_{g}\right)\right\| \zeta^{T} \zeta \\
& \leq\left(g a_{\mathrm{nc}}^{\prime}+a_{\phi}\right) \zeta^{T} \zeta=a_{\mathrm{nc}} V
\end{aligned}
$$

where $a_{\mathrm{nc}}^{\prime}:=2\left\|\left(D(\ell) \otimes P^{-1}\right) H_{i}\right\|, a_{\phi}$ is the positive constant introduced above, and $a_{\mathrm{nc}}=\left(g a_{\mathrm{c}}^{\prime}+a_{\phi}\right) / \underline{\lambda}$.

Equation (24) means that, in general, $V(\zeta)$ is increasing when disconnected topologies occurs.

\section{Hybrid analysis}

We now proceed towards the proof of Proposition 2 by considering the network of agents under switching topologies. We will consider the Lyapunov function (22) and analyze the behavior of the agents during connected flows, disconnected flows and jumps of topology.

First we estimate the jump in the value of $V(\zeta)$ when a change in the topology occurs, namely when $\mathcal{T}_{i+1}$ replaces $\mathcal{T}_{i}$. Denoting by $\zeta^{+}$and $\chi^{+}$the "next value" of the state variables $\zeta$ and $\chi$ when a jump in the topology occurs, we note that $\chi^{+}=\chi$ and

$$
\zeta^{+}=J_{i}^{+} \chi^{+}=J_{i+1} \chi=J_{i+1} J_{i}^{-1} \zeta
$$

Hence, by letting

$$
\bar{v}=\max _{i, j \in\left[1, \ldots N_{\mathrm{t}}\right]}\left\|J_{j} J_{i}^{-1}\right\|
$$

we can easily bound the jump of the Lyapunov function when the topology switches as

$$
\begin{aligned}
V^{+} & =\zeta^{+T}\left(D(\ell) \otimes P^{-1}\right) \zeta^{+} \leq \bar{\lambda}\left\|\zeta^{+}\right\|^{2} \\
& \leq \bar{\lambda} \bar{v}^{2}\|\zeta\|^{2} \leq \frac{\bar{\lambda}}{\underline{\lambda}} \bar{v}^{2} V:=a_{\mathrm{j}} V .
\end{aligned}
$$

We will continue the analysis by considering the closed-loop networked system as an hybrid system flowing during the time intervals in which the communication topology is connected ( $i$ even), and "instantaneously" jumping in the intervals in which the topology is disconnected. To this end, let $i$ be even and let $t_{i}, t_{i+1}$ be, respectively, the times at which the topology switches from $\mathcal{T}_{i} \in \mathcal{T}_{\mathrm{c}}$ to $\mathcal{T}_{i+1} \in \mathcal{T}_{\text {nc }}$, and from $\mathcal{T}_{i+1}$ to $\mathcal{T}_{i+2} \in \mathcal{T}_{\mathrm{c}}$. By bearing in mind (24) and (26), we have that the jump undergone by the Lyapunov function between two connected topologies can be estimated as

$$
\begin{aligned}
V\left(t_{i+1}^{+}\right) & \leq a_{\mathrm{j}} V\left(\zeta\left(t_{i+1}^{-}\right)\right) \leq a_{\mathrm{j}} e^{a_{\mathrm{nc}} T_{0}} V\left(\zeta\left(t_{i}^{+}\right)\right) \\
& \leq a_{\mathrm{j}} e^{a_{\mathrm{nc}} T_{0}} a_{\mathrm{j}} V\left(\zeta\left(t_{i}^{-}\right)\right)=e^{\sigma_{\mathrm{j}}} V\left(\zeta\left(t_{i}^{-}\right)\right)
\end{aligned}
$$

with $\sigma_{\mathrm{j}}:=a_{\mathrm{nc}} T_{0}+2 \ln \left(a_{\mathrm{j}}\right)$.

We are thus left to study an hybrid system governed by (23) during flows and instantaneously jumping as $V^{+} \leq e^{\sigma_{\mathrm{j}}} V$, with the length of the flow intervals governed by an average dwell time of the form (16).

The fact that the time intervals satisfy an average dwell-time condition given by (16) allows one to say (see [2]) that flow and jump times of the hybrid system can be thought of as governed by a clock variable $\varsigma$ flowing according to the differential inclusion $\dot{\varsigma} \in[0,1 / \tau]$ when $\varsigma \in\left[0, n_{0}\right]$ and jumping as $\varsigma^{+}=\varsigma-1$ when $\varsigma \in\left[1, n_{0}\right]$. 
We thus endow the networked system with the clock variable and study the resulting hybrid system whose Lyapunov function flows and jumps according to the following rules

$$
\begin{aligned}
& \left.\begin{array}{rl}
\dot{\zeta} & \in[0,1 / \tau] \\
\dot{V} & \leq-a_{\mathrm{c}} V
\end{array}\right\} \quad(\varsigma, V) \in\left[0, n_{0}\right] \times \mathbb{R} \\
& \left.\begin{array}{rl}
\varsigma^{+} & =\varsigma-1 \\
V^{+} & \leq e^{\sigma_{\mathrm{j}}} V
\end{array}\right\} \quad(\varsigma, V) \in\left[1, n_{0}\right] \times \mathbb{R}
\end{aligned}
$$

Specifically, following [2], let

$$
\mathcal{W}(\varsigma, \zeta)=e^{L \varsigma} V(\zeta)
$$

with $L \in\left(\sigma_{\mathrm{j}}, \tau a_{\mathrm{c}} / 2\right)$. During flows, by compactly writing (20) as $\dot{\zeta}=F\left(\zeta, z_{1}\right)$, we have that for all $v \in$ $\operatorname{col}\left([0,1 / \tau], F\left(\zeta, z_{1}\right)\right)$

$$
\begin{aligned}
\langle\nabla \mathcal{W}(\tau, \zeta), v\rangle & =L e^{L \varsigma} \dot{\zeta} V(\zeta)+e^{L \varsigma}\left\langle\nabla V(\zeta), F\left(\zeta, z_{1}\right)\right\rangle \\
& \leq L e^{L \varsigma} \frac{1}{\tau} V(\zeta)-a_{\mathrm{c}} e^{L \varsigma} V(\zeta) \\
& \leq-\left(a_{\mathrm{c}}-\frac{L}{\tau}\right) \mathcal{W}(\varsigma, \zeta) \\
& \leq-\frac{a_{\mathrm{c}}}{2} \mathcal{W}(\varsigma, \zeta)
\end{aligned}
$$

for all $\left(\varsigma, \zeta, z_{1}\right) \in\left[0, n_{0}\right] \times \mathbb{R}^{\left(N_{\mathrm{a}}-1\right) d} \times \mathbb{R}^{d}$. On the other hand, during jumps, we have that

$$
\begin{aligned}
\mathcal{W}^{+} & =e^{L \varsigma^{+}} V\left(\zeta^{+}\right) \leq e^{L(\varsigma-1)} e^{\sigma_{\mathrm{j}}} V(\zeta) \\
& =e^{-L+\sigma_{\mathrm{j}}} e^{L \varsigma} V(\zeta)=e^{-L+\sigma_{\mathrm{j}}} \mathcal{W}(\varsigma, \zeta) \\
& =\varepsilon \mathcal{W}(\varsigma, \zeta)
\end{aligned}
$$

with $\varepsilon=e^{-L+\sigma_{\mathrm{j}}} \in(0,1)$. The Lyapunov function $\mathcal{W}(\cdot, \cdot)$ is thus decreasing both during flows and during jumps. This and the fact that $\mathcal{W}$ is positive definite with respect to the set $\left[0, n_{0}\right] \times\{0\}$ lead to the conclusion that the set $\left[0, n_{0}\right] \times\{0\}$ is globally asymptotically stable. This, by taking advantage from Assumption 2 and from the cascade structure of system (20)-(21), proves the result.

\section{Simulation Results}

To verify the result provided in Section III and in Section IV, two numerical frameworks are presented. In the first scenario five Lorentz oscillators are taken into account and a fixed disconnected topology is considered. In the second, the same set of agents is considered under switching topology.

\section{A. Lorentz Oscillators Clustering}

The Lorentz oscillators are described by

$$
\begin{aligned}
& \dot{x}_{k_{1}}=\sigma\left(x_{k_{2}}-x_{k_{1}}\right) \\
& \dot{x}_{k_{2}}=x_{k_{1}}\left(\rho-x_{k_{3}}\right)-x_{k_{2}} \quad y_{k}=x_{k_{1}} . \\
& \dot{x}_{k_{3}}=x_{k_{1}} x_{k_{2}}-\beta x_{k_{3}}
\end{aligned}
$$

for $k=1, \ldots, 5$. The values of parameters $(\sigma, \rho, \beta)$ are $\sigma=10, \rho=28$ and $\beta=8 / 3$. System (27) can be embedded into the fourth order system (see [17] for further details)

$$
\begin{aligned}
& \dot{w}_{k_{1}}=w_{k_{2}} \\
& \dot{w}_{k_{2}}=w_{k_{3}} \\
& \dot{w}_{k_{3}}=w_{k_{4}} \\
& \dot{w}_{k_{4}}=\Phi\left(w_{k_{1}}, w_{k_{2}}, w_{k_{3}}, w_{k_{4}}\right)
\end{aligned}
$$

fitting into the structure of (5) and fulfilling the assumption listed in Section II-B.

In new coordinates, the agents' initial conditions are $w_{1}=(1.5 ; 1 ; 0 ; 0), w_{2}=(1 ; 5 ; 5 ; 5), w_{3}=(2 ; 10 ; 10 ; 10)$, $w_{4}=(0.5 ; 7 ; 7 ; 7)$ and $w_{5}=(0 ; 15 ; 15 ; 15) . K$ is chosen as in $(8)$ with the gain parameter $g=50$. 

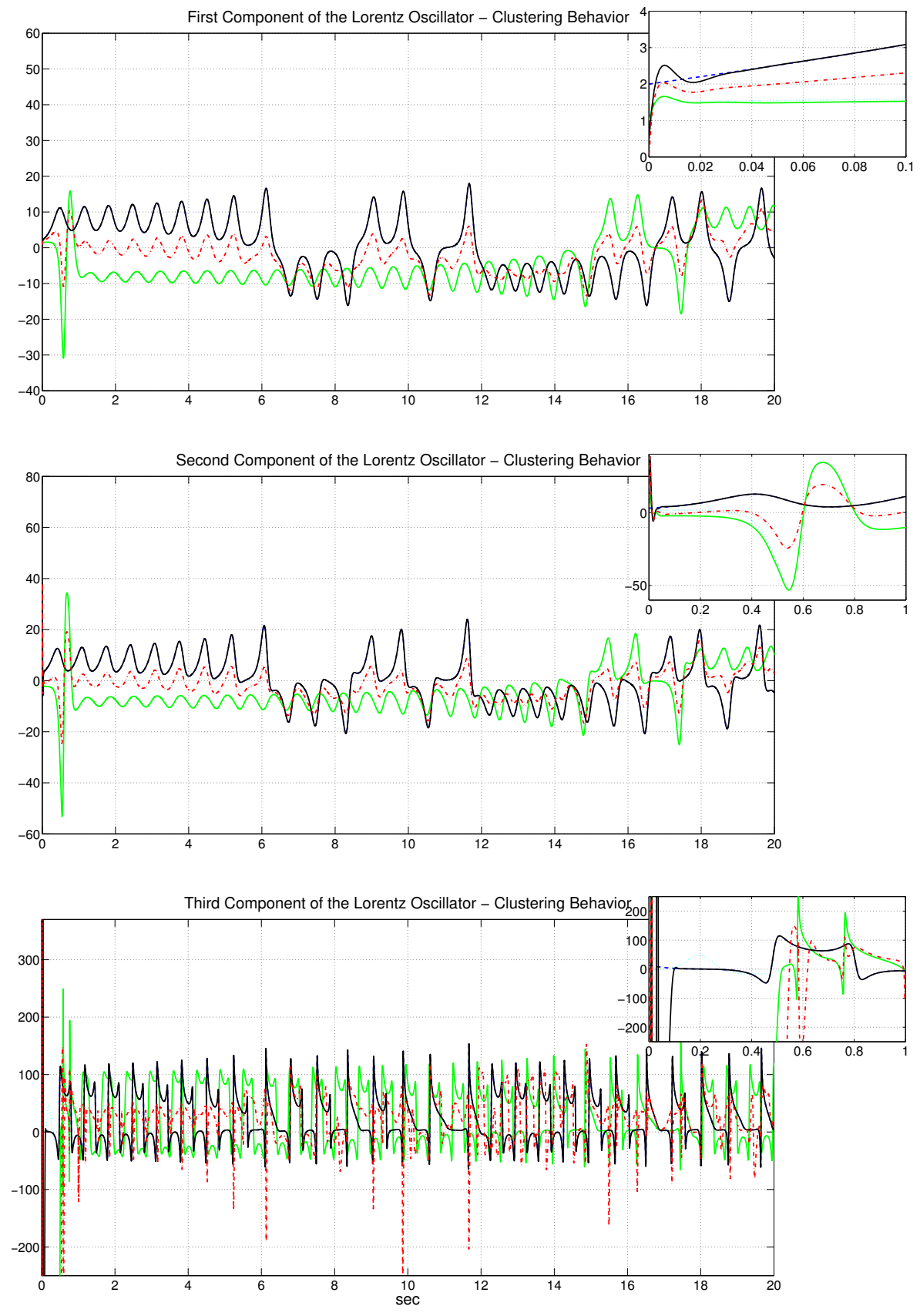

Fig. 1. Lorentz oscillators with disconnected topology: the agents belonging to the two $I C C$ s achieve consensus while the residual agent (red dotted signal) is simply bounded.

The fixed disconnected topology considered in this example is given by the Laplacian matrix

$$
L=\left[\begin{array}{ccccc}
0 & 0 & 0 & 0 & 0 \\
-1 & 1 & 0 & 0 & 0 \\
0 & 0 & 0 & 0 & 0 \\
0 & 0 & -1 & 1 & 0 \\
-1 & -1 & -1 & -1 & 4
\end{array}\right]
$$

It is trivially seen that this topology is composed by two $I C C$ : the first one is $I C C_{1}=\left\{w_{1}, w_{2}\right\}$, while the second is $I C C_{2}=\left\{w_{3}, w_{4}\right\}$. Agent $w_{5}$ is the residual component of the graph and receives information from all the other clusters. 
Figure 1 shows the behavior of the system in the original coordinates (27). As expected from the analysis developed in Section III, the agents belonging to the two ICC achieve clustered consensus (the black line and the green line in the plot), while the residual agent (the red dotted line) is simply bounded.

\section{B. Lorentz Oscillators Synchronization}

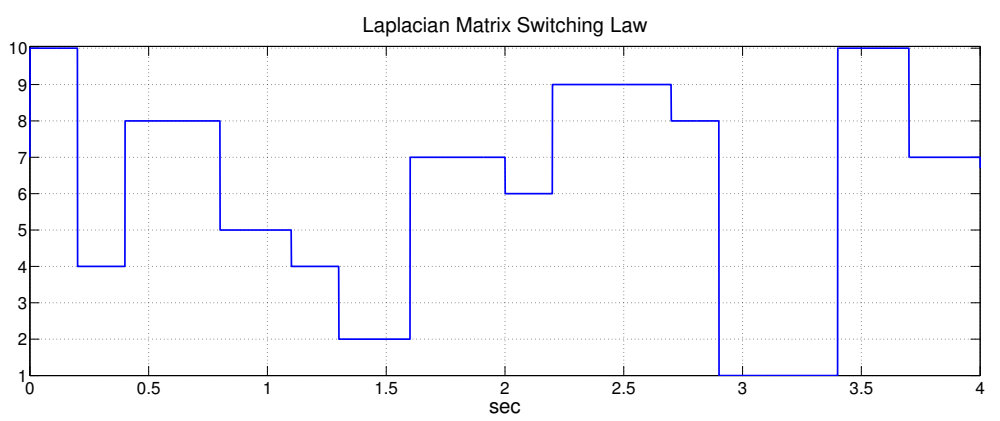

Fig. 2. Switching topology for Lorentz oscillator: $4 \mathrm{sec}$ of switching law are shown, fulfilling the requested limitation in terms of $T_{0}$, $N_{0}$ and $\delta$.

In order to show simulation results in case of switching topology, we selected ten random topologies (odd disconnected, even connected), with $T_{0}=0.3 \mathrm{sec}, N_{0}=3$ and $\delta=2$ (see Figure 2 for a sample of $4 \mathrm{sec}$ ). The control parameter $K$ is chosen as in (8) with the gain parameter $g=50$.

Figure 3 shows the three components of the five Lorentz oscillators in the original coordinates (27) achieving synchronization. In the top right corner, the zoom shows the initial transient towards synchronization.

\section{CONCLUSIONS}

In this paper we considered the problem of homogeneous network of agents and disconnected topologies. In particular we showed that, in presence of disconnected topologies, the diffusive coupling design proposed in [18] (namely high gain design) does not impact on the stability of the agents. Furthermore, we showed that agents belonging to independent connected components achieve consensus, giving rise to a clusterization of the agents. The residual agents, not belonging to any of the independent connected components, are instead bounded.

In the second part of the paper, we addressed the problem of achieving consensus within a network of homogeneous nonlinear agents with switching communication (connected and disconnected) topologies. We have proved that if the switching rule of connected topologies fulfills an average dwell-time condition consensus is achieved. The proposed result considers a network of homogeneous agents. Following the arguments in [18], however, robust consensus among heterogeneous nonlinear systems exchanging information within a switching network of the kind considered in this paper can be obtained.

The idea and the Lyapunov tool used in the paper to prove the main result are, in principle, useful in other networked control contexts that can be subject of investigation. For instance, a possible improvement is to weaken the graph connectivity assumption considered here by just asking joint connectivity with the different topologies required to fulfill a certain dwell-time only in the average. Furthermore, a possible extension is to adapt the analysis we developed to a fully hybrid scenario, where each agent and local controller are hybrid, rather than continuous-time systems.

\section{REFERENCES}

[1] R. Goebel, R. Sanfelice, A. Teel, "Hybrid Dynamical Systems", Princeton University Press.

[2] C. Cai, A.R. Teel, R. Goebel, "Smooth Lyapunov functions for hybrid systems, Part II: (Pre)-asymptotically stable compact sets", IEEE Trans. Trans. on Automatic Control, 53, pp. 734-748, 2008.

[3] B. Nabet, N. E. Leonard, I. D. Couzin and S. A. Levin, "Dynamics of Decision Making in Animal Group Motion", Journal of Nonlinear Science, 19, pp. 399-435, 2009.

[4] I. C. Morarescu and A. Girard, "Opinion dynamics with decaying confidence: application to community detection in graphs", IEEE Trans. on Automatic Control, 56(8), 1862-1873, 2011. 

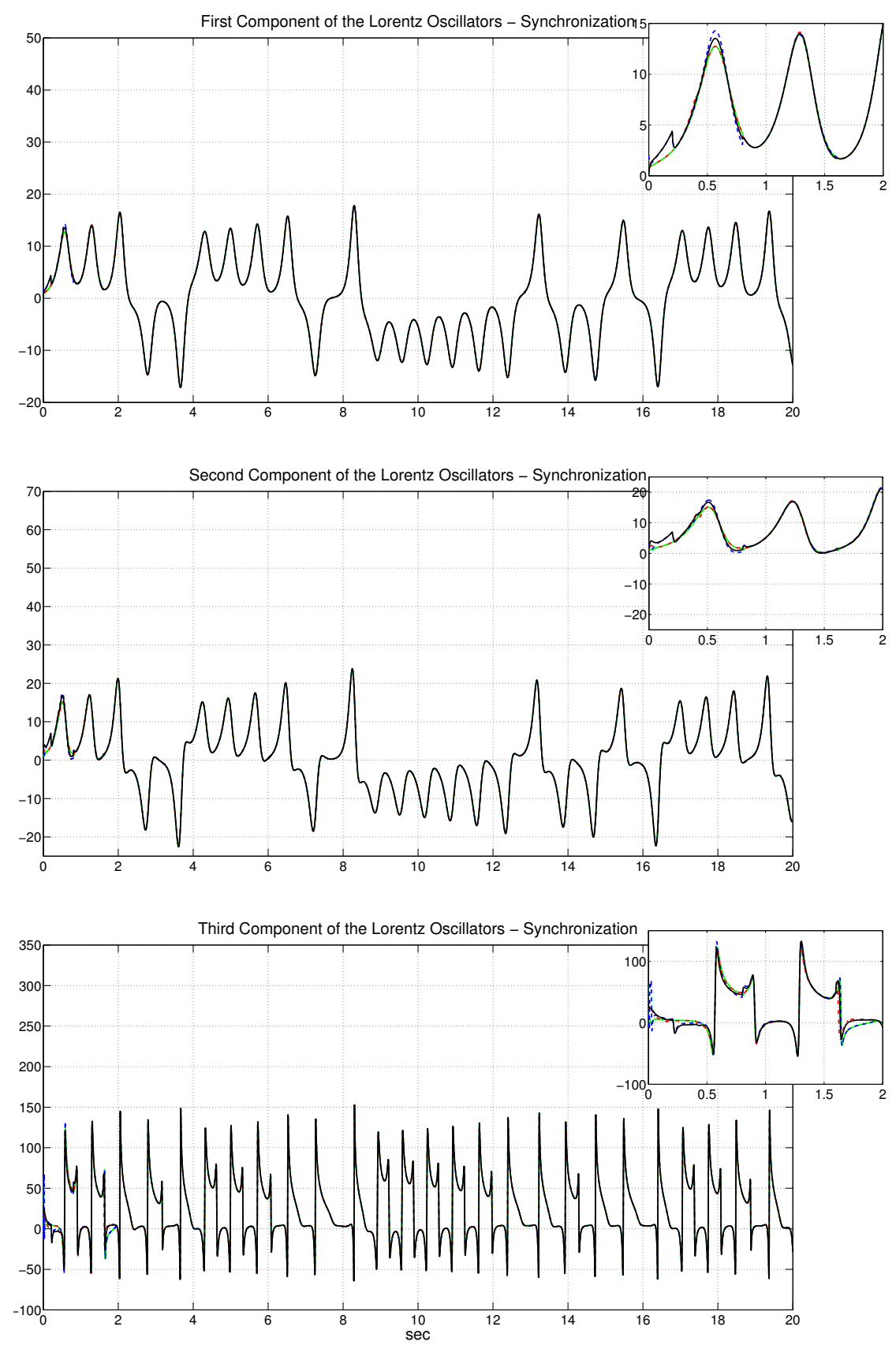

Fig. 3. Lorentz oscillators reaching consensus: the three components of the Lorentz oscillators are shown. Small zoom in the top right corner shows the transient behavior of the oscillators achieving consensus.

[5] G. Shi and Y. Hong, "Global target aggregation and state agreement of nonlinear multi-agent systems with switching topologies", Automatica, 45, pp. 1165-1175, 2009.

[6] T. Yang, Z. Meng, G. Shi, Y. Hong and K. H. Johansson, "Synchronization for Multi-agent Systems under Directed Switching Topologies", arXiv:1401.6541v1, 25 Jan 2014.

[7] Qiang Jia and Wallace K. S. Tang, "Consensus of Nonlinear Agents in Directed Network With Switching Topology and Communication Delay", IEEE Trans. on Cicrcuits and Systems, 59(12), pp. 3015-3023.

[8] T. Yang, Z. Meng, G. Shi, Y. Hong and K. H. Johansson, "Network Synchronization with Nonlinear Inherent Dynamics and Switching Interactions", arXiv:1401.6541v2, 29 Dec 2014.

[9] J. Zhao, D. J. Hill, T. Liu, "Synchronization of complex dynamical networks with switching topology: A switched system point of view", Automatica, 45, 2502-2511, 2009.

[10] S. Li, Y. Guo, "Distributed Consensus Filter on Directed Graphs with Switching Topologies", American Control Conference, 2013.

[11] U. Munz, A. Papachristodoulou and F. Allgower "Consensus in Multi-Agent Systems With Coupling Delays and Switching Topology", 
IEEE Trans. on Automatic Control, 56(12), pp. 2976-2982, 2011.

[12] R. Olfati-Saber, R. M. Murray, "Consensus Problems in Networks of Agents with Switching Topology and Time-Delays", IEEE Trans. on Automatic Control, 49(9), pp. 1520-1533, 2004.

[13] L. Moreau, "Stability of multi-agent systems with time-dependent communication links", IEEE Trans. on Automatic Control, 50(2), pp. 169-182, 2005.

[14] N. Monshizadeh, H. L. Trentelman and M. K. Camlibel, "Uniform synchronization in multi-agent systems with switching topologies", accepted onInternational Journal of Robust and Nonlinear Control, 2015.

[15] P. Wieland, From Static to Dynamic Couplings in Consensus and Synchronization among Identical and Non-Identical Systems, PhD thesis, Universität Stuttgart, 2010.

[16] P. Wieland, R.Sepulchre and F. Allgöwer. "An internal model principle is necessary and sufficient for linear output synchronization". Automatica, 47, 1068-1074, 2011.

[17] F. Forte, L. Isidori and A. Isidori, "Robust Design of Internal Models by Nonlinear Regression", 9th IFAC Symposium on Nonlinear Control Systems, pp. 283-288, 2013.

[18] A. Isidori, L. Marconi and G. Casadei, "Robust Output Synchronization of a Network of Heterogeneous Nonlinear Agents Via Nonlinear Regulation Theory", IEEE Trans. on Automatic Control, 59(10), pp. 2680-2692, 2014.

[19] G. Casadei, C. De Persis and L. Marconi, "About disconnected topology and cluster consensus", 1st IFAC MICNON, St. Petersburg, July 2015.

[20] G. Casadei, L. Marconi and A. Isidori, “About synchronization of homogeneous nonlinear agents over switching networks", IEEE Conf. on Decision and Control, pp. 4573-4578, 2014.

[21] E. D. Sontag "A Remark on the Converging-Input Converging-State Property", IEEE Trans. on Automatic Control, 48(2), pp. 313-314, 2003.

[22] J. P. Hespanha and A. S. Morse, "Stability of switched systems with average dwell-time", IEEE Conf. on Decision and Control, pp. 2655-2660, 1999.

[23] C. Cai, A. R. Teel and R. Goebel Smooth Lyapunov Functions for Hybrid Systems Part II: (Pre)Asymptotically Stable Compact Sets. IEEE Trans. on Automatic Control, 53( 3), pp. 734-748, 2008

[24] D. Liberzon, "Switching in Systems and Control", Birkhauser, Boston, MA, Jun 2003. Volume in series Systems and Control: Foundations and Applications.

[25] C. Godsil, G. Royle, "Algebraic Graph Theory", Graduate Texts in Mathematics, 207, Springer.

[26] W. Ren and R. W. Beard, "Consensus seeking in multiagent systems under dynamically changing interaction topologies", IEEE Trans. on Automatic Control, 50(5), pp. 655-661, 2005.

[27] F. Dorfler and F. Bullo, "Synchronization and transient stability in power networks and non-uniform Kuramoto oscillators", American Control Conference, pp. 930-937, 2010.

[28] D. J. Hill and G. Chen, "Power systems as dynamic networks, IEEE Int. Symposium on Circuits and Systems, pp. $722-725,2006$.

[29] S. Trip, M. Burger and C. De Persis, "An internal model approach to frequency regulation in power grids", http://arxiv.org/abs/1403.7019.

[30] X. Zhang and A. Papachristodoulou, "Distributed Dynamic Feedback Control for Smart Power Networks with Tree Topology", American Control Conference, pp. 1156-1161, 2014.

[31] A. Isidori, "Nonlinear Control Systems", Springer Verlag, 1995.

[32] W. E. Roth, "The equations $A X-Y B=C$ and $A X-X B=C$ in matrices", Proc. American Mathematical Society, 3, pp. 392-396, 1952. 